\section{Use of a general analysis of variance program in missing data situations}

\author{
JOHN F. WALSH \\ Fordham University, Bronx, New York 10471
}

Procedures are described which enable researchers to easily modify a general $\mathrm{N}$-way analysis of variance program so that it can be used in unequal $\mathrm{N}$ cases. Advantages in terms of range of application, storage requirements, and accuracy are presented. FORTRAN instructions illustrating the general approach are given.

Most computer-center libraries contain a general $\mathrm{N}$-way analysis of variance program. A usual requirement of such a program is that there be equal Ns in each of the cells. This feature is frequently impossible to meet in a considerable number of research situations. The $\mathrm{E}$, then, is usually left with the options of getting additional data, eliminating cases until the largest common multiple among the cell sizes is reached, or following a "cookbook" analysis for unweighted means on a desk calculator. All of these are joyless prospects.

While there may be a computer program for unweighted means for a specific design in a center, it is clearly desirable to have a direct and easily coded procedure for implementing this technique for a wide range of balanced designs. The computations can be divided broadly into three parts (Hemmerle, 1967). The first part is calculation of the analysis of variance as if it were a standard analysis with one score per cell. These calculations are the largest single computational portion. Use can be made of existing programming codes to compute this first part. The additional two parts of the calculations are computation of the error term, which is based upon individual data elements, and development of the harmonic mean of the cell sizes. The programming that is required to obtain these latter two quantities is direct.

The material below describes the implementation for a particular example. The structure of the programming permits easy extrapolation to different balanced designs. To obtain the three quantities indicated above, an input section of coding instructions and three subroutines-Avdat, Avcal, and Meanq-from IBM's scientific subroutine package (1969) are required. These subroutines implement the general decomposition algorithm developed by Hartley (1967). In addition to the arrays required by these subroutines, the user supplies three. Temp and Xbar are singly subscripted arrays. Their sizes are number of levels corresponding to that factor in the experiment.

To illustrate the procedure, Table 1 contains the arrays and the FORTRAN code for the input part of a 2 by 3 factorial experiment with disproportionate cell frequencies, the largest being 20 . These cell frequencies are stored in the array Ncell and are read in at execution time, along with the values of the limits of the factors, viz, II and JJ. The ordering of the sizes of the cell frequencies on this initial card reflects the sequencing in the DO loops controlling the statement that reads the data cards. The number and order of these DO loops reflect the sorting procedure used to prepare the data for input. Specifically, the data is sorted on Ss initially, then on the variable associated with the I subscript, then on the variable associated with the $J$ subscript. This sorting pattern differs from the SSP procedure where equal Ns are required. The code computes the cell means (Xbar), the sum of squares within cells (Sserr), the degrees of freedom for error (Ndferr), and the harmonic mean (Hmean). The variable Ndata is used to index the cell means

TABLE 1

FORTRAN CODE FOR INPUT OF A TWO-WAY ANALYSIS OF VARIANCE

DIMENSION LEVEL (2), ISTEP(2), KOUNT (2), MSTEP(2), LASTS(2)

DIMENSION NDF( 3$), \operatorname{SMEAN}(3), \operatorname{SUMSQ}(3), \operatorname{NCELL}(2,3), X B A R(12)$

DIMENSION TEMP(20)

DATA NDATA, HMEAN, SSERR, TN $/ 0,3 \div 0.1$

$\operatorname{READ}(1,2)$ II , J J, ((NCELL $(I, J), I=1, I I), J=1\rfloor J$,

2 FORMATC $2 I 1,6 I 2)$

DO $20 \mathrm{~J}=1, \mathrm{~J} J$

DO 20 I $=1$, I I

NDATA $=$ NDATA +1

$X B A R(N D A T A)=0$.

$N N=N C E L L(1, j)$

HMEAN=HMEAN+1./NN

$T N=T N+N N$

DO $15 L=1, N N$

$15 \operatorname{READ}(1,11) \operatorname{TEMP}(L)$

C ENTER APPROPRIATE FORMAT CARD HERE

DO $10 L=1, N N$

$10 \times B A R(N D A T A)=X B A R(N D A T A)+T E M P(L)$

$X B A R(N D A T A)=X B A R(N D A T A) / N N$

DO $5 L=1, N N$

5 SSERR $=S S E R R+(T E M P(L)-X B A R(N D A T A)) \% 2$

20 CONTINUE

HMEAN=NDATA/HMEAN

NDFERR $=T N-I I \% J \mathrm{~J}$

ERR $=S S E R R /(H M E A N * N D F E R R)$

LEVEL $(1)=$ I I

LEVEL $(2)=J$ J

$K=2$

CALL AVDAT (K, LEVEL, NDATA, XBAR, L, ISTEP, KOUNT)

CALL AVCAL ( $K$, LEVEL, XBAR, L, ISTEP, LASTS)

CALL MEANQ (K, LEVEL, XBAR, GMEAN, SUMSQ, NDF, SMEAN, MSTEP, KOUNT, 1 LASTS)

C FORM F RATIOS AND OUTPUT THE SUMMARY TABLE 
as they are stored linearly in the array $\mathrm{Xbar}$. This storage mode extends the usefulness of the routines by including those versions of FORTRAN which limit the number of subscripts to three or four. With linear representation, the analysis of $\mathrm{N}$-way designs is limited only by the storage capacity of the user's computer center.

The computed cell means are passed as an argument to the sequence of subroutines listed above. The user need only assign values to the arguments, $\mathrm{K}$, and the array level. As mentioned previously, $K$ equals the number of factors in the analysis, and the array level contains the limits of the number of categories of each factor. The other arguments required by the routines are either variables or arrays defined by the subroutines themselves or have been computed during the input portion of the program.

Meanq returns a complete analysis of variance based upon means. To produce a scaled estimate of error to be used with the values returned from Meanq, Hmean is divided into Sserr (Meyers, 1966). F ratios for main effects and interactions are then computed. As an alternative, one could scale the values returned by Meanq upward by multiplying each by Hmean and computing $F$ ratios, using the computed Sserr directly as the estimate of error (Winer, 1962).

The procedure outlined above has a number of features that should be emphasized. Initially, such a program can be used for equal, proportionate, and disproportionate cell sample sizes. Analysis of unweighted means for the equal and proportionate cases yields exact $F$ ratios, so the $E$ does not sacrifice accuracy by basing the analysis on means rather than on individual data points. In addition, the storage requirements are reduced dramatically from the situation where the analysis uses the individual data elements.

For example, the size of the array needed to accommodate the data points for a $3^{5}$ factorial with 15 scores per cell, using the three subroutines mentioned earlier, is 16,384 locations, with each location requiring 4 bytes of core storage. In contrast, the procedure outlined above, using means, would require 1,024 locations for the cell means, plus 15 additional ones for the cell elements.

In general, analysis based on the individual data points will require $\mathrm{N}$ times more storage than an analysis based upon unweighted means. Such a difference in core storage requirements for two procedures that yield equivalent answers in two of three situations is an important consideration. This is particularly the case for Es having access to small computers such as IBM's 1130 series. For the case where the cell sizes are disproportionate, the use of unweighted means is the E's only realistic option.

Finally, the computational scheme used in the subroutines in implementing Hartley's algorithm is based on deviations from cell totals. This procedure minimizes the likelihood that high-order digits will be lost in the subtract magnitude operation during intermediate computations. Consequently, the results returned by the program are expected to have good relative error.

\section{REFERENCES}

HAR'TLEY, H. O. Analysis of variance. In A. Ralston and H. Wilf (Eds.) Mathematical methods for digital computers. New York: Wiley, 1967. Pp. 221-230.

HEMMERLE, W. J. Statistical computations on a digital computer. Waltham: Ginn Blaisdell, 1967.

IBM Scientific Subroutine Package. White Plains: IBM, 1969. Manual H 20-0205-3. MEYERS. J. Fundamentals of experimental design. Boston: Allyn \& Bacon, 1966.

WINER, B. J. Statistical principles in experimental design. New York: McGraw-Hill, 1962.

\title{
Using a general analysis of variance algorithm for covariance designs
}

\author{
JOHN F. WALSH \\ Fordham University, Bronx, New York 10458
}

Procedures are described which enable researchers to implement balanced covariance designs of from one to four independent variables. Use is made of three subroutines from IBM's Scientific Subroutine Package which implement a general decomposition algorithm for balanced designs. FORTRAN instructions, illustrating the main calling program, are given.

Application of the analysis of covariance technique by behavioral scientists in research areas is extensive. The combination of interest in the adjustments provided plus the considerable computational requirements of the procedure have made this program one of the first to appear in computer-center libraries. Usually, however, library programs such as the biomedical series (BMD) from the University of California (Dixon, 1970) require large amounts of core and are not easily modifiable by the user. One runs them as is, or else does the analysis by hand.

The present article is intended for researchers with small-to-modest computer configurations. The material below describes procedures which permit persons with modest knowledge of FORTRAN to implement covariance designs quite easily. Use is made of three subroutines-AVDAT, AVCAL, and MEANQ-from the Scientific Subroutine Package (SSP) (IBM, 1969). These routines implement the general decomposition algorithm developed by Hartley (1962).

Table 1 presents the FORTRAN code for the main calling program. The arrays are set to accommodate designs involving from one to four independent variables with $\mathrm{N}$ Ss per cell. In determining the array sizes, space was provided for the inclusion of Ss as a pseudofactor. Specifically, the arrays are set to accommodate four independent variables plus the pseudofactor of Ss. The size of each of the arrays, $M$, for the covariate $X$, variate $Y$, and crossproduct variable $\mathrm{XY}$, is computed as follows:

$$
\mathbf{M}=\prod_{\mathrm{i}=1}^{\mathrm{K}}[\operatorname{LEVEL}(\mathrm{I})+1],
$$

where LEVEL(I) = number of levels of the factor and $K=$ number of factors, including Ss.

On the first input card, the user punches the required parameters in 12 format. The parameters are the number of factors, $K$, and the number of categories or levels in each of them. The levels of the pseudofactor $S$ are listed last. The FORTRAN variable names for the factors are II, JJ, LL, $\mathrm{MM}$, and NS, respectively. If less than four independent variables are involved, the value of 1 is entered for those factors not included in the design. For example, if the design being analyzed involved a 3 by 4 analysis with $10 \mathrm{Ss}$ per cell, the first 